\title{
Research on BDC Mental State Model for the Command Mechanism
}

\author{
Cao Bowei \\ Academy of Armored Forces Engineering \\ AAFE \\ Beijing, China \\ caoboweinwu@163.com \\ Zhang Guohui \\ Academy of Armored Forces Engineering \\ AAFE \\ Beijing, China
}

\begin{abstract}
The simulation of the various behavior process of the combat units is an important part of combat simulation, and the behavior of combat units needs driving from the mental state process of the combat units. So, based on the study of Agent cognitive model based on the theory in BDI, and in order to overcome the shortcoming of operational applications in combat, this paper comes up with the more suitable for military operations BDC mental state model with command centre and achieved with "command" instead of "intent" as the core of the mental state to discuss the characteristics of the individual mind - CABC logical characteristics.
\end{abstract}

Keywords-Combat units, BDC model,

Instruction mechanisms, Mental state model

\section{INTRODUCTION}

In recent years, combat simulation has become important means to army combat simulation training, weapons and equipment design as well as shape and assessment. In the course of combat, Agent-oriented instruction is mainly reflected in the characteristics of mechanisms to realize their own intention. It is also need to consider the joint intention of groups to ensure coordination requirements between groups and individual. In combat, the special nature of the military system, determining the collaboration and group intent is mainly passed and realized by combat instruction. Command mechanism can fully reflect the wishes and intentions of commanders' with high priority and mandatory, and typically reflect the characteristics of military operations [1].

There are some defects in the traditional BDI model in CABC study. This paper is not the intention of the traditional center and replaced by military system better able to express and easy to grasp the thinking property - orders, instructions to create a mechanism to express characteristics of the operations for Agent social logic.

\section{MEASURING AUTOBODY PANELS}

\section{A. Intention and Command}

As far as Agent-BDI model was concerned, not only show that how intention was produced from beliefs, desires and other mental state, but also that intended to produce in

\author{
Xue Qing \\ Academy of Armored Forces Engineering \\ AAFE \\ Beijing, China \\ xue_qing@yeah.net \\ Wei Yang \\ PLA General Armament Department 33 department \\ Xi'An, China
}

the planning and implementation role in the phenomenon associated with the group.

Comparison of the two concepts of intention and command, showing that individuals tend to prefer intension is a desire for their own individual targets; and command is a group of concepts, reflected in the other individual to individual requirements and aspirations of a is individual aspirations and a commitment to the group. Command can actually be seen as Agent for others cherished intention. Mental state in the BDI model, when the Agent wish to achieve a state, it committed to the implementation of their own that can achieve the status of the action series. The basic characteristics of the individual intention is to perform action of its "self-commitment."

From the general sense, the intention of more significance is the thinking of individual properties, is difficult to speculate from the outside the intention of an Agent of the pregnant, and different Agent also may have different intentions logic system, the intent is still the philosophical and psychological nature Studies have not yet fully resolved. The intention does play an important role in human activity, but it is not fit for describe the Agent's activities with the centre of intention in the CABC research. In contrast, command does describe the restraint relation between individual instruction, or other individual and group constraints. It is typical of the social thinking attributes. Command is from the group of other individuals (commanders) of the collaborative requirements, reflecting the desire and intent of commanders with high priority and mandatory. This command relationship is easy to grasp from the outside, but also easy to Agent's own reasoning, and thus suitable for military applications CABC research.

Instruction is the choice of future behavior, because the mandatory directive mechanism, the instruction and intention there is a big difference. Command has the following basic features:

(1) Agent's instructions and constraints will affect the future behavior of Agent;

(2) Instruction is able to be satisfied, Agent will not accept the theorem that can not be satisfied as its command, or Agent will give up those instructions have not been met;

(3) Agent must be consistent between the instruction, that Agent can not simultaneously be processed on the 
directive are inconsistent, inconsistent instructions Agent will not know how to act in accordance with its instructions;

(4) Agent of the instruction is consistent with the belief, Agent should not be both a command, at the same time that the instruction is not satisfied;

(5) Agent shall not conflict between the instruction that the Agent is not a command to stop or hinder the realization of another command successful. But the number of Agent' $s$ instructions may conflict with each other;

(6) Command is persistent, that Agent will not be free to abandon its existing instructions, instruction Agent with a changing environment in the continuous ownership of the instructions. Agent sustained success is to achieve its directive is an important condition.

B. Agent for the hierarchical structure of command mechanisms

Document [2] take for that, $\mathrm{CABC}$ is a social individual, their mental state property should reflect the social. Therefore, it can be divided CABC into groups layer and individual layer. The mental state can be further divided into layers of social consciousness and individual consciousness level.

From Layer in the individual consciousness, CABC only in the sense of individual belief, desire and intention memory and processing attributes of thinking, although some mental states involved in other Agent, before the rise to social awareness level it has no effect on other Agent. Therefore, belief, desire, intention and goals of thinking element mode attribute is a word, one Agent to another Agent's beliefs, desires and intentions can be naturally attributed to the corresponding element model.

Level of social consciousness, Agent through instruction, commitment, and other social attributes of memory and thinking, reasoning, build relationships with other Agent. Thinking attributes this layer with multiple layers of individual consciousness Agent-related support groups are also the basic properties of the state level. So, can the order dealing with such a binary mode. In the CABC, the command is the driving force Agent acts, directives into specific behavioral objectives of the course, but faith is the knowledge about the instruction and the target.

Instruction in a group layer, joint intention, collective commitment and normative groups such phenomena can be attributed to a combination of individual layer properties. Group thought to be attributed to the individual properties of the combination of mental states, groups of social commitment is considered the "glue", the joint intention is to organize the core of the concept of collective activities, instruction is the use of force intended to convey the group, reflecting the $\mathrm{CABC}$ in the level and hierarchy, is the basic form of Agent Collaboration. Agent's commitment to the performance of the targets is individual level instruction.

Therefore, in the $\mathrm{CABC}$, Agent model has three basic and most important attributes of thinking, a layer of individual consciousness element mode - Belief and desire, social awareness of the dual layer mode - - command. This state of mind these three attribute combinations as BDC model to command the center to discuss the agent's mental state features.

\section{DATA PROCESSING}

\section{A. BDC model structure}

In the highly dynamic battlefield environment, the need to combat perception of the battlefield Agent changes in the environment and the dynamic changes depending on the environment to adjust their goals and intentions in order to drive the implementation of appropriate action. BDCbased model structure, Agent's internal mental state can be beliefs, desires and attributes such as explicit instructions to that fight in the combat environment Agent's deliberative process, this paper presents a model based on the internal structure of BDC. The model is mainly composed of Knowledge storage, Beliefs library.

Knowledge storage required to achieve a variety of problem-solving knowledge, such as the side of the operational doctrines, operational principles. The quality and completeness of the knowledge base determines the extent of the intelligence level of CABC. Among them, the situation assessment knowledge, the information is used to explain the perception, classification, formation of belief; scenario - desire to match the rules for the results of perception (belief) of reasoning, the formation of desire; decision space, including the formation of intentions from a variety of aspirations knowledge; success stories can speed up the reasoning that if the current trend is the $\mathrm{CABC}$ has successfully gone through, then extracted directly from the success stories in the case, without having to repeat the reasoning; behavior space knowledge, including how to target high-level behavior into a series of element such knowledge, and a variety of element behavior algorithm, called conditions. In addition, the external environment, acquaintances and other factual information but also as a model of knowledge stored in the Knowledge Base, which means that the system acquaintance with one's own related entities, is generally lower and the neighbor on the side, the side face of the enemy and so on. Acquaintance model determines the scope and perception of the main objects and interactive way.

Beliefs library stores the information from CABC'S understanding and judgments to external environment and other CABC. It is that including intentions to all the attributes of battlefield environment, attributes of enemy situation, the neighboring state properties as well as belief in a specific target on the battlefield. Intention is defined as the accumulation of existing evidence. Therefore, the conviction of a property for each database value is the current belief in the faith from the previous time the perception of value and the current time value (or interact with the results obtained $\mathrm{CABC}$ ) jointly determined.

Rao and Georgeff [3] as the desire to set the task, or achieve the desired goal may be the main path set. Rao and Georgeff this article on the desire to follow the definition, and therefore wishes library stores $\mathrm{CABC}$ possible path to achieve the desired objectives 
set. These may be the path set is the CABC library based on faith belief that the value of the current situation in the use of knowledge - desire reasoning produced matching rules.

Desire to focus all desire the same goals is tied, the final CABC will perform one of them. So, it is derived based on local information. Therefore, under the specific situation, some wish it easy to implement, requires a high price and some desire to achieve; may have the opportunity to realize some desire, some may never be realized. It is the final decision of $\mathrm{CABC}$-making behavior through an optimal choice from a desire to focus on the desire to act with intent (instructions), and through the implementation of the directive to achieve this goal.

Instruction library storing the behavior plan of $\mathrm{CABC}$, that is to achieve the desired goal of commitment to the implementation. Perception is engaged in every moment, thus the evolving objectives and to achieve the target instruction. These instructions are on target or priority in the order form is stored in the command library. Instruction library by undertaking to achieve the successful implementation of the final instructions, to give up due to changes in the external environment can not continue to execute instructions.

Wishes and instructions are on one of the main events in the state of hope, their difference is: desire is possible path to achieve their goals, and instruction is possible paths in the optimal path, the instruction will guide and control the main future activities. The same objectives, the relationship between desire and command options is similar to decision analysis and optimal solutions in the relationship.

Instruction can be seen as part of the behavior of programs, which are the main objectives in order to achieve its commitment to the implementation of the plan. The most obvious is the nature of instruction will lead to behavior that the command is the behavior of the controller. To sum up, the primary role of instruction includes that as following:

(1) Command to control the subject of ongoing behavior;

(2) Directive of the deliberative process of the future (or target selection);

(3) order-driven scenarios of the future - aspirations match reasoning;

(4) Instruction of the future practical reasoning is based on faith.

\section{$B$. BDC model of the mechanism of instruction}

$\mathrm{CABC}$ receiving stimuli from the environment or other body of information, its interpretation and classification, and store it in the belief that library. Because the information environment, many can not accept every kind of information and processing, therefore, can acquaintance model, only accept their own goals related to environmental and other subject information. External information is that information of natural environment and through the sensor to detect the enemy status information. Because perception is engaged in all the time, creating a sense sequence, the current perception of the information may be different from the previous perception of the information, then we should update the belief base is the perceived behavior of the thinking activity. As the main information from other external events including the parent's instructions, requests for the same level or lower as well as consult information.

Scenes are stored in the Knowledge Base - matching rules desire, kept aware of knowledge input and knowledge base to search, matching and reasoning, the scene when a match or receive instructions, request information, trigger matching rules, have aspirations set. Desire to describe the feelings of $\mathrm{CABC}$ preferences, wishes can be with the current (or initial) instruction compatible, but also allow the existence of unreachable aspirations. Then consider the need to keep the current instruction and commitment to get with the current goal (corresponding to cope with external threats or respond to a request) and up to the aspirations of compatible subset (ex, the target set), and desire into the library. Despite the desire to desire to set goals can be related with the current, but due to limited resources, CABC was not possible to pursue all the goals. At this time need to achieve the objective of price or ease, CABC their ability level and preferences to filter the principles, drawn desire to complete the objectives as the moment the best instruction, and instruction into the library.

\section{OUTLOOK}

Based on the classic shortcomings of BDI model based on the proposed mechanism in the military command, $\mathrm{CABC}$ layer should be divided into groups, and individual level, while the individual Agent's mental state layer should also be used include the sense of social consciousness level and individual level hierarchical model, so as to better reflect the individual's social.

Intended for the BDI model for instruction in the weakening of the environment, this paper presents a new mental state model,BDC, use the Command instead of Intention as the core of Agent mental state of individuals to discuss the CABC characteristics. Non-standard modal logic as a formal tool used to study the Agent and the BDC model, formally defined the concept of the instruction, and instruction as a mental state property and the belief, desire and other organic combination. As a reflection of the level of instruction and compulsory features state of mind, in the Agent layer hierarchical model of social consciousness, reflecting the population of other individuals in a group for the Agent desire and intention, is the Agent to reach the state of commitment in the future. The model solves the agent with intent to cause too much emphasis on the behavior of self-confusion, for the $\mathrm{CABC}$ technology laid the theoretical foundation

However, because there is no belief in BDC model operator for any extended defects such as [4], therefore, logical omniscience problem still exists. This is also the need for further discussion and study.

\section{REFERENCES}

[1] Simon Shiu, Sankar K Pal. Case-Based Reasoning: Concepts, Features and Soft Computing. Applied Intelligence, November 2004,21 (3): 233-238

[2] Wei Yang. Agent based instruction system of combat modeling and simulation [D], PhD thesis Armored Force Engineering Institute, 2010.06 
[3] Rao A S, Georgeff M P. BDI Agente: from theory to practice. Proceedings of the 1 st International Conference on Multi-Agent Systems (ICMAS-95). San Francisco, 1995: 312-319

[4] Elizabeth M Biddle. Multiplayer Computer Games: A Team Performance Assessment Research and Development Tool.
Proceeding of the 9thConference on Computer Generated Forces and Behavioral Representation. Orlando, Florida, America, May, 2008:76-83 